\title{
Measuring the dynamics of circular dichroism in a pump-probe experiment with a Babinet-Soleil Compensator
}

\author{
Claire Niezborala and François Hache \\ Laboratoire d'Optique et Biosciences, Ecole Polytechnique, CNRS-INSERM, 91128 Palaiseau cedex, France
}

Received May 26, 2006; revised July 26, 2006; accepted August 4, 2006; posted August 17, 2006 (Doc. ID 71350)

Circular dichroism contains rich information on the conformation of molecules and, in particular, of biomolecules, and measuring its variation in a pump-probe experiment is very promising but also very challenging. We propose a new technique to measure pump-induced variation of the circular dichroism, which is based on the measurement of the probe ellipticity and its variation with the pump. This technique has the advantage that it does not require modulation of the probe polarization, which allows modulation of the pump intensity. We show theoretically and demonstrate experimentally that this technique is very sensitive and user friendly. We also show that it can be used to measure pump-induced change in the optical rotation, allowing for a complete characterization of pump-induced optical activity. (C) 2006 Optical Society of America

OCIS codes: $260.2130,300.6500$.

\section{INTRODUCTION}

Circular dichroism [(CD): difference in absorption for a left- and a right-circularly polarized light] is known to be a very powerful probe of the molecule conformations. In large molecules and especially in biomolecules, it mostly originates from the coupling between the various constituents and is therefore sensitive to their relative positions. Static CD is routinely used, in particular, to elucidate the secondary structure of proteins. Extension to time-resolved spectroscopy is very appealing because such a technique could give information on the dynamics of the conformation of proteins, which is a major issue of biophysics. Pioneering work in this direction was carried out by Lewis et al. ${ }^{1}$ and Xie and Simon, ${ }^{2}$ who obtained time-resolved CD signals in carbonmonoxymyoglobin with $7 \mathrm{~ns}$ and $50 \mathrm{ps}$ resolutions, respectively, showing that the deoxymyoglobin form was instantaneously reached on these time scales. We recently extended this experiment to a subpicosecond time resolution ${ }^{3}$ and obtained a sub$100 \mathrm{ps}$ dynamics, which we assigned to a deformation of the proximal histidine following the heme doming. ${ }^{4}$ Even though the experimental details are somewhat different, all these experiments are based on the same principle: a classical pump-probe setup is used, and one measures the $\mathrm{CD}$ as a function of the pump-probe delay. In Ref. 1, the probe is made slightly elliptical by a strained plate, whereas in Refs. 2 and 3, it is made circular. In every case, the CD measurement techniques rely on the modulation of the probe polarization. Modulation of the circular polarization from left to right is achieved by a photoelastic modulator ${ }^{2}$ or a Pockels cell. ${ }^{3}$ These techniques are straightforward, but they have several drawbacks. First, they are prone to many artifacts. The most important one comes from the pump-induced linear dichroism, which can yield a fake signal if the two modulated polarizations are not perfectly symmetrical. ${ }^{5}$ To correct this problem, we have developed a stringent alignment procedure of the
Pockels cell. ${ }^{6}$ The second drawback is that these techniques are not very sensitive to the pump-induced change. $C D$ is naturally a very weak signal $\left(\sim 10^{-3}\right.$ compared to the absorption in biomolecules), and it is often difficult to measure its variation in a pump-probe experiment due to technical fluctuations. The problem is that the usual pump-modulation techniques, which are currently used to improve the signal-to-noise ratio in pumpprobe experiments are not easy to implement because of the probe polarization modulation.

In this paper, we propose a new scheme to measure the pump-induced variation of the CD. In this scheme, we keep the probe linearly polarized and measure the ellipticity induced by the sample with a Babinet-Soleil (BS) compensator combined with a crossed analyzer. Actually, this setup is usually not precise enough to measure the steady-state CD of the sample, but things are quite different when one is interested in the change of CD induced by a pump pulse. Modulating the pump intensity with a mechanical chopper, we find that the modulated part in the transmitted signal is very sensitive to the ratio of the induced CD to the induced absorption change. For samples and spectral range where the change in CD is dominant compared to the change of absorption, this technique proves to be very robust and yields relevant information much more easily than the usual probe polarization modulation technique. This feature is especially interesting in the far UV where the secondary structures of proteins ( $\alpha$ helices, $\beta$ strands, etc.) present characteristic CD spectra, although their absorption spectra are identical. ${ }^{7}$

In Section 2, we investigate this technique theoretically and derive the relevant equations. These equations allow us to propose an experimental procedure providing easy measurement of pump-induced CD change. Sensitivity and noise limitations are also discussed. This procedure is demonstrated in the Section 3 on a chiral ruthenium complex and applied to the photolysis of carbonmonoxymyo- 
globin and its subsequent CD change at $265 \mathrm{~nm}$. Full demonstration of the potentiality of this technique is given. We also show that tuning the analyzer instead of the BS compensator gives a direct measurement of the pump-induced rotation polarization, optical rotation $(\mathrm{OR})$, allowing for a complete characterization of pump-induced optical activity to be performed with this technique.

\section{THEORY}

\section{A. Probe Alone: Jones Matrix Analysis}

We consider the experimental setup depicted in Fig. 1. A linearly polarized beam is sent through the sample and then is passed through a BS compensator and an analyzer. Two parameters are accessible to the experiment: the BS phase retardation and the analyzer angle. These two parameters can be independently adjusted so that, in the absence of a sample, the transmitted intensity is null. If one inserts a sample that is nonchiral and free of birefringence, no change in the transmission is observed. On the contrary, if the sample is chiral, it displays optical activity (OR and CD), which perturbs the transmission. To analyze the effect of the various parameters, we develop a Jones matrix calculation. ${ }^{8}$ Let $(x, y)$ be the laboratory frame, $x$ (respectively, $y$ ) corresponding to the vertical (respectively, horizontal) direction. The incoming laser field is

$$
E^{\text {in }}=E_{0}\left[\begin{array}{l}
1 \\
0
\end{array}\right]
$$

Each element on the beam path can be described by a 2 $\times 2$ matrix, and the output beam reads as

$$
E^{\text {out }}=M_{\text {Analyzer }} M_{\mathrm{BS}} M_{\text {Sample }} E^{\text {in }} .
$$

$M_{\text {Sample }}$ is the matrix describing the sample taking optical activity into account. Its calculation follows the one given in Ref. 9. Let us first define the relevant parameters. Let $n_{L}, n_{R}, \alpha_{L}, \alpha_{R}$ be, respectively, the refractive indices and the absorption coefficients for the left and right circular polarization. We define the OR as

$$
\delta=2 \pi \frac{n_{L}-n_{R}}{\lambda} L
$$

and the CD as

$$
\eta=\left(\alpha_{L}-\alpha_{R}\right) L
$$

The matrix $M_{\text {sample }}$ then reads as

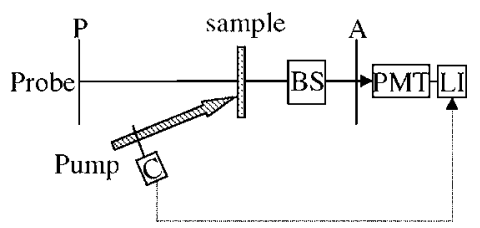

Fig. 1. Scheme of the experimental setup. P, polarizer; A, analyzer; BS, Babinet-Soleil compensator; PMT, photomultiptier tube; LI, lock-in amplifier; C, mechanical chopper.

$$
M_{\text {Sample }}=e^{(-\alpha L / 2)}\left[\begin{array}{cc}
\cosh \left(\frac{\eta}{4}+\mathrm{i} \frac{\delta}{2}\right) & -\mathrm{i} \sinh \left(\frac{\eta}{4}+\mathrm{i} \frac{\delta}{2}\right) \\
\mathrm{i} \sinh \left(\frac{\eta}{4}+\mathrm{i} \frac{\delta}{2}\right) & \cosh \left(\frac{\eta}{4}+\mathrm{i} \frac{\delta}{2}\right)
\end{array}\right],
$$

where $\alpha=\left(\alpha_{L}+\alpha_{B}\right) / 2$ is the mean absorption coefficient (in intensity) and $L$ is the sample thickness. This matrix takes CD and circular birefringence into account. These two effects commute and can be considered together without trouble. ${ }^{9}$

We suppose that the BS axes are oriented at $45^{\circ}$ compared to the laboratory frame. Calling $2 X$ its retardation, its matrix is

$$
M_{\mathrm{BS}}=\left[\begin{array}{cc}
\cos X & \mathrm{i} \sin X \\
\mathrm{i} \sin X & \cos X
\end{array}\right] .
$$

Finally, denoting $\varepsilon$ as the analyzer angle (measured with respect to the crossed position), $M_{\text {Analyzer }}$ reads as

$$
M_{\text {Analyzer }}=\left[\begin{array}{cc}
\sin ^{2} \varepsilon & \sin \varepsilon \cos \varepsilon \\
\sin \varepsilon \cos \varepsilon & \cos ^{2} \varepsilon
\end{array}\right] .
$$

From Eq. (1), it is straightforward to obtain $E^{\text {out }}$. However, the total expression is cumbersome, and we prefer to use an approximate expression. Effects of optical activity are usually very weak and $\delta$ and $\eta$ are very small numbers. The relevant $X$ and $\varepsilon$ are therefore also very small numbers. We choose to keep only the lowest nonzero order in these numbers, which yields

$$
E^{\text {out }}=E_{0} e^{(-\alpha L / 2)}\left[\left(\varepsilon-\frac{\delta}{2}\right)+\mathrm{i}\left(X+\frac{\eta}{4}\right)\right],
$$

or, in terms of intensity;

$$
I^{\text {out }}=e^{-\alpha L}\left[\left(\varepsilon-\frac{\delta}{2}\right)^{2}+\left(X+\frac{\eta}{4}\right)^{2}\right] .
$$

We can analyze this last equation. If there is no sample (or if the sample is nonchiral), $I^{\text {out }}=0$ for $\varepsilon=0$ and $X=0$. Changing the analyzer angle (respectively, the BS retardation) yields a parabola versus $\varepsilon$ (respectively, versus $X$ ). When a chiral sample is inserted, these two parabolas shift. The parabolas now have their minima away from zero. The shifts directly give $\delta$ and $\eta$. This technique to measure the OR and the CD is, however, not very efficient because it demands a very high precision on the position of the parabola minima, which is very challenging. We will see now that this limitation is lifted in a pump-probe experiment if one wants to access the variation of the OR and CD.

\section{B. Pump-Probe Experiment}

When a pump beam is sent onto the sample, this results in a change of absorption, CD, and OR. Let $\alpha_{0}, \delta_{0}$, and $\eta_{0}$ be the values when the pump is off, and $\Delta \alpha, \Delta \delta$, and $\Delta \eta$ their variations due to the pump. The measured intensity without the pump then reads as 


$$
\begin{aligned}
I_{\text {without pump }} & =e^{-\alpha_{0} L}\left[\left(\varepsilon-\frac{\delta_{0}}{2}\right)^{2}+\left(X+\frac{\eta_{0}}{4}\right)^{2}\right] \\
& \equiv e^{-\alpha_{0} L}\left[\rho^{2}+Y^{2}\right] .
\end{aligned}
$$

With the new variables $\rho$ and $Y$, the intensity in presence of the pump is

$$
I_{\text {with pump }}=e^{-\alpha_{0} L} e^{-\Delta \alpha L}\left[\left(\rho-\frac{\Delta \delta}{2}\right)^{2}+\left(Y+\frac{\Delta \eta}{4}\right)^{2}\right] .
$$

Let us now consider the experimental case where the pump is modulated by a mechanical chopper. Two quantities are readily measurable: the mean output intensity, hereafter called the PM signal, which is the average of $I_{\text {with pump }}$ and $I_{\text {without pump }}$ and the modulated part of the PM signal obtained with a lock-in amplifier, which we call the LI signal equal to $I_{\text {with pump }}$ and $I_{\text {without pump. From }}$ Eqs. (9) and (10), we obtain

$$
\begin{aligned}
\mathrm{PM}= & \frac{e^{-\alpha_{0} L}}{2}\left[\rho^{2}\left(e^{-\Delta \alpha L}+1\right)-\rho \Delta \delta e^{-\Delta \alpha L}+\frac{1}{4} \Delta \delta^{2} e^{-\Delta \alpha L}\right. \\
& \left.+Y^{2}\left(e^{-\Delta \alpha L}+1\right)+\frac{1}{2} Y \Delta \eta e^{-\Delta \alpha L}+\frac{1}{16} \Delta \eta^{2} e^{-\Delta \alpha L}\right], \\
\mathrm{LI}= & e^{-\alpha 0 L}\left[\rho^{2}\left(e^{-\Delta \alpha L}-1\right)-\rho \Delta \delta e^{-\Delta \alpha L}+\frac{1}{4} \Delta \delta^{2} e^{-\Delta \alpha L}\right. \\
& \left.+Y^{2}\left(e^{-\Delta \alpha L}-1\right)+\frac{1}{2} Y \Delta \eta e^{-\Delta \alpha L}+\frac{1}{16} \Delta \eta^{2} e^{-\Delta \alpha L}\right] .
\end{aligned}
$$

These expressions can be now utilized to analyze the signals obtained when varying the experimental parameters $\rho$ and $Y$. Let us consider the case where the analyzer angle is fixed ( $\rho$ is kept constant, not necessarily null), and let us investigate the variation of the PM and LI signals as a function of the BS retardation $Y$. We obtain

$$
\begin{aligned}
& \mathrm{PM}=\frac{e^{-\alpha_{0} L}}{2}\left[Y^{2}\left(e^{-\Delta \alpha L}+1\right)+\frac{1}{2} Y \Delta \eta e^{-\Delta \alpha L}+K_{1}\right], \\
& \mathrm{LI}=e^{-\alpha_{0} L}\left[Y^{2}\left(e^{-\Delta \alpha L}-1\right)+\frac{1}{2} Y \Delta \eta e^{-\Delta \alpha L}+K_{2}\right] .
\end{aligned}
$$

In these equations, the $K_{i}$ 's are constant (with respect to $Y$ ). This will also be true of the forthcoming $K_{i}$ 's. Because these constants do not play a role in the discussion, we will not give their expressions. From Eqs. (13) and (14), one can see that the PM and LI versus $Y$ curves are parabolas, as expected at this level of approximation. The important point here is to notice that these two parabola do not have the same minima position nor the same curvature. To make this clear, we normalize the LI parabola to the PM one by defining the BS variable as

$$
Z=Y+\frac{\Delta \eta e^{-\Delta \alpha L}}{4\left(e^{-\Delta \alpha L+1}\right)} .
$$

Note that passage from $X$ to $Y$ and to $Z$ is merely a shift of the origin but does not involve change of the scale. Dividing now PM and $\mathrm{LI}$ (which are known only to within the same multiplicative constant) by $\left(e^{-\alpha_{0} L} / 2\right)\left(e^{-\Delta \alpha L}+1\right)$, we obtain

$$
\begin{gathered}
\mathrm{PM}=Z^{2}+K_{3}, \\
\mathrm{LI}=2 \frac{e^{-\Delta \alpha L}-1}{e^{-\Delta \alpha L}+1} Z^{2}+\Delta \eta \frac{2 e^{-\Delta \alpha L}}{\left(e^{-\Delta \alpha L}+1\right)^{2}} Z+K_{4} .
\end{gathered}
$$

From these expressions, one clearly sees that comparing the PM and LI parabolas directly yields $\Delta \alpha$ and $\Delta \eta$. In the case where $\Delta \alpha L \ll 1$, the LI signal reduces to

$$
L I=-\Delta \alpha L Z^{2}+\frac{1}{2} \Delta \eta Z+K_{8}
$$

which shows that the curvature of the LI parabola gives $\Delta \alpha L$, whereas the minimum shift yields $\Delta \eta$.

\section{Discussion}

From the previous calculation and especially from Eqs. (16)-(18), one can see that plotting the PM signal and the LI signal on the same graph as a function of the BS retardation gives two parabola with different curvatures and minimum positions. The change in curvature directly yields the pump-induced absorption; it exists for all samples. On the contrary, one sees that the two parabola will be shifted only for chiral samples that display a pump-induced CD change. The important point here is that this shift is equal to $\Delta \eta / 4 \Delta \alpha$. Therefore, if the change in absorption $\Delta \alpha$ is weak, this ratio can be quite large, resulting in an easily measurable shift. This will be demonstrated in the next section. Another advantage of this technique is that it can be readily extended to the complementary case where the BS retardation is kept fixed (preferentially but not necessarily at $X=0$ ), and the PM and LI curves are plotted as functions of the analyzer angle. In that case, Eqs. (16)-(18) can be utilized again by changing $\Delta \eta$ by $\Delta \delta$. All the conclusions on the measurement of the pump-induced CD are therefore valid for the measurement of the pump-induced OR. It is worthwhile noticing that these two determinations are independent of one another and that uncertainty on one measurement does not invalidate the other one.

This new technique has several advantages compared to the usual one where the probe polarization is modulated. First, as already stated, it allows modulation of the pump intensity, which strongly increases the sensitivity of the measurement, not only because of the already discussed intrinsic enhancement when $\Delta \alpha$ is weak, but also because of the strong signal-to-noise increase inherent to pump-modulation techniques. Second, only linearily polarized beams impinge on the sample. This has two favorable consequences. It eliminates many artifacts connected to a bad symmetry between the probe polarizations. ${ }^{5,6}$ Furthermore, it allows for the magic angle configuration to be used. Let us recall that this configuration consists of sending the pump and the probe with an angle of $54.7^{\circ}$ in order to get rid of parasitic orientational effects. ${ }^{10}$ This was not possible with the other techniques. Utilizing the 
magic angle configuration, however, has a drawback because it can induce linear birefringence and dichroism in the sample that perturbs the $\mathrm{CD}$ and $\mathrm{OR}$ measurement (see Appendix A).

Before concluding this section, it is worth discussing the effects of noise in these measurements. As already stated, the pump-modulation technique allows one to be much less sensitive to the laser fluctuations. However, it is very difficult to eliminate residual electronic offsets in the PM and LI signals (due, for example, to drift in the boxcar signal or electromagnetic radiation). Actually, these offsets must be included in the $K_{i}$ 's in the above equations. They do not perturb the measurements, which do not rest on the absolute values of the signals but only on the shape of the parabolas. The only noise that can perturb the measurement is the scattering of the pump because, being polarized and modulated, this noise would appear as a fictitious $\Delta \alpha$. Fortunately, such a noise is usually easy to eliminate. In fact, the main uncertainty comes from the degree of precision in the determination of the parabola minima. A quantitative example is given in Section 3 .

In conclusion, we think that this new technique is very promising because it yields relevant signals with an increased sensitivity, and it allows complementary measurements (CD and OR) to be readily performed. Its only limitation is that it necessitates samples with a high CDchange-to-absorption-change ratio and is not applicable if the absorption change is too strong. In the next section, we will demonstrate its capabilities in a UV experiment in carbonmonoxymyoglobin, which fulfills is the required conditions.

\section{EXPERIMENTAL DEMONSTRATION}

\section{A. Direct Proof with a Chiral Ruthenium Complex}

To experimentally check the conclusions of the previous section, we have performed a pump-probe experiment in a chiral ruthenium-tris(phenanthroline) salt (RuTP). Three samples were available: the racemic mixture and the two enantiomers ( $\Lambda$ and $\Delta$ ). In each case, a $150 \mu \mathrm{M}$ solution in acetonitrile was placed in a $1 \mathrm{~mm}$ thick silica cell. The setup conforms to the sketch in Fig. 1. The sample is placed between a polarizer and a crossed analyzer (extinction ratio of $10^{-5}$ ). A BS compensator is inserted between the sample and the analyzer. A mechanical chopper is placed on the pump path; the modulation is at $80 \mathrm{~Hz}$. The polarizations of the pump and the probe are parallel. The probe beam transmission is measured by a photomultiplier tube, and the signal is integrated by a boxcar (PM signal) and a lock-in amplifier locked on the chopper modulation frequency (LI signal). Retardation of the BS and angle of the analyzer are precisely computer controlled with stepper motors. The time resolution of this setup, obtained from the pump-probe correlation (see below), is $\sim 800 \mathrm{fs}$. The probe wavelength is tuned at $265 \mathrm{~nm}$. This wavelength was chosen because it corresponds to prominent CD features in the ruthenium complex as well as for myoglobin. This pulse is generated by several stages of optical parametric amplification starting from a $1 \mathrm{kHz}$ Ti:sapphire laser, and its duration is $\sim 700 \mathrm{fs}$ and its energy is in the nanojoule range. The pump beam

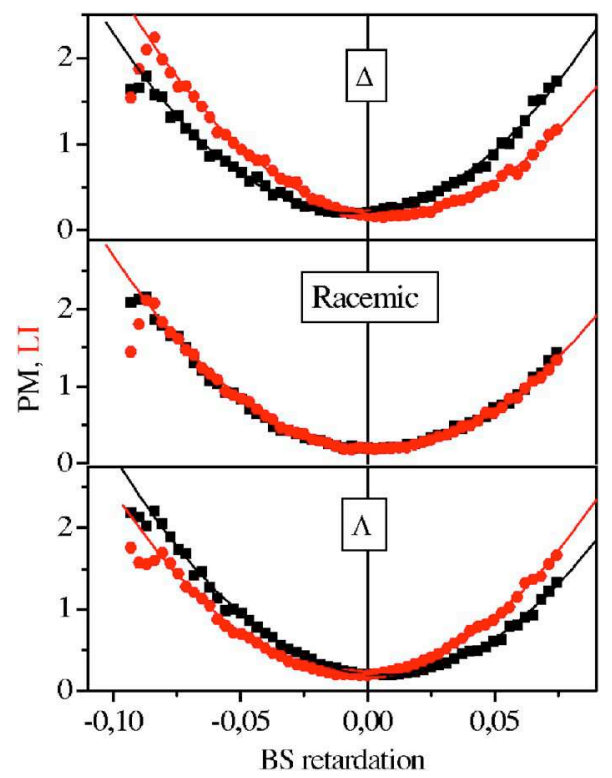

Fig. 2. (Color online) PM (squares) and LI (dots) signals in arbitrary units as a function of the BS retardation (in radian) for the $\Delta$ and $\Lambda$ enantiomers and for the racemic mixture. The sign of the LI curves has been changed to allow for an easier comparison of the parabola.

is directly obtained by frequency doubling the Ti:sapphire pulses. Its energy is a few hundreds of nanojoules. Pump and probe beams are focused with $250 \mathrm{~mm}$ focal length lenses with an angle of $4^{\circ}$.

When photoexcited at $400 \mathrm{~nm}$ in the metal-to-ligand charge transfer band, RuTP displays an absorption bleaching in the ligand $\pi-\pi^{*}$ transition at $265 \mathrm{~nm}$. To measure the pump-induced CD, we maintain the analyzer in the crossed position and vary the BS retardation. Following the procedure of Section 2, we have measured the output intensity as a function of the BS retardation for a fixed positive pump-probe delay. Raw results are displayed in Fig. 2 for the three RuTP samples. For each sample, the PM and LI curves are plotted in arbitrary units. It is clear that for the racemic RuTP sample, the two parabolas have their minima at the same position; whereas the two minima are strongly shifted for the enantiomers. This is in agreement with our expectations: the two parabola have distinct minima only for a chiral sample. These curves are rich in information. First of all, considering only the PM curves, one can observe symmetrical shifts between the two enantiomers and the racemic mixture. These small but noticeable shifts allow for the measurement of the steady-state CD of RuTP: $\eta_{0}$ $=2.4 \pm 0.1 \times 10^{-2}$ corresponding to $\Delta \varepsilon=700 \mathrm{M}^{-1} \mathrm{~cm}^{-1}$. Second, the LI curves yield the pump-induced absorption and CD changes. Here again, as expected for a chiral signal, the shifts between the LI and PM curves are symmetrical for the two enantiomers. This symmetry ensures that the observed shift is a consequence of the chirality of the sample and cannot be due to artifacts. Note that, on the contrary, the curvatures are the same, indicating that the (achiral) absorption change is the same for the three samples. From the PM and LI parabola, it is straightforward to extract $\Delta \alpha$ and $\Delta \eta$ from Eqs. (16)-(18), and we deduce $\Delta \alpha L=3.3 \times 10^{-2}$ and $\Delta \eta=+2.8 \times 10^{-3}$ for the $\Lambda$ enan- 
tiomer and $\Delta \eta=-3.8 \times 10^{-3}$ for the $\Delta$ one. The difference in the measurements comes from the aforementioned uncertainty on the parabola minima. In our experiment, this uncertainty is estimated to $5 \times 10^{-3} \mathrm{rad}$, leading to an uncertainty of $7 \times 10^{-4}$ for $\Delta \eta$. This uncertainty could be reduced by a finer tuning of the BS retardation and by averaging several curves. We have also checked that when the pump intensity is increased, the position of the minimum of the LI curve does not change, and that both absorption and CD changes depend linearily on the pump intensity.

\section{B. Application to the Photolysis of Carbonmonoxymyoglobin}

As an illustration of this technique, we have considered the photolysis of carbonmonoxymyoglobin. Myoglobin is a hemoprotein, which can reversibly fix ligands such as $\mathrm{O}_{2}$, $\mathrm{CO}$, or NO. The fixation site is the "heme" cofactor. The heme has a strong absorption in the visible, and it is well established that its photoexcitation induces the departure of the ligand and an instantaneous change of its electronic state. ${ }^{11}$ Myoglobin, like most of the proteins, is a chiral molecule whose optical activity lies in the coupling of the various amino acids and of the heme. The CD spectra of the liganded form $(\mathrm{MbCO})$ and of the deoxy form $(\mathrm{Mb})$ are displayed in Fig. 3. Sending a visible pulse on the sample allows one to pass from one spectrum to the other one. The strong structure in the visible is related to the heme absorption. We have already thoroughly studied its dynamics. ${ }^{3,4}$ This spectral region is, however, not adapted to our new technique, because the change in the electronic structure of the heme is accompanied by a strong variation of the absorption. On the contrary, the structure at $\sim 260 \mathrm{~nm}$ presents good characteristics. We have recently studied pump-induced absorption in this region and found that it is very weak. This feature can be related to the fact that in this spectral range, there is a strong contribution of the aromatic amino acids, which are not sensitive to the visible pump. On the contrary, as can be seen in Fig. 3, passage from $\mathrm{MbCO}$ to $\mathrm{Mb}$ generates a strong variation of the $\mathrm{CD}$. Probing of $\mathrm{MbCO}$ photolysis at $265 \mathrm{~nm}$ therefore perfectly fulfils the condition for the optimal use of our new technique. The sample is a

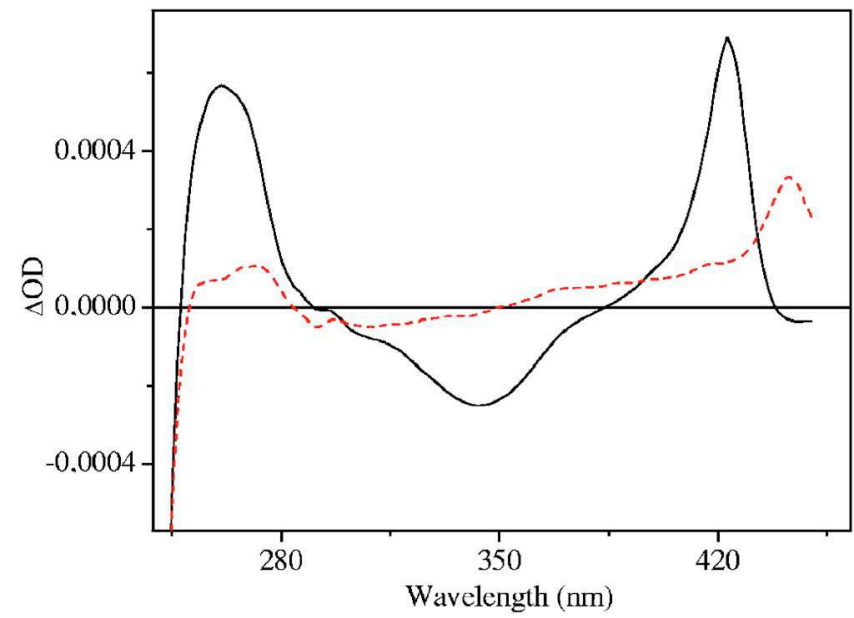

Fig. 3. (Color online) $\mathrm{CD}$ spectra for $\mathrm{MbCO}$ (solid curve) and $\mathrm{Mb}$ (dashed curve).

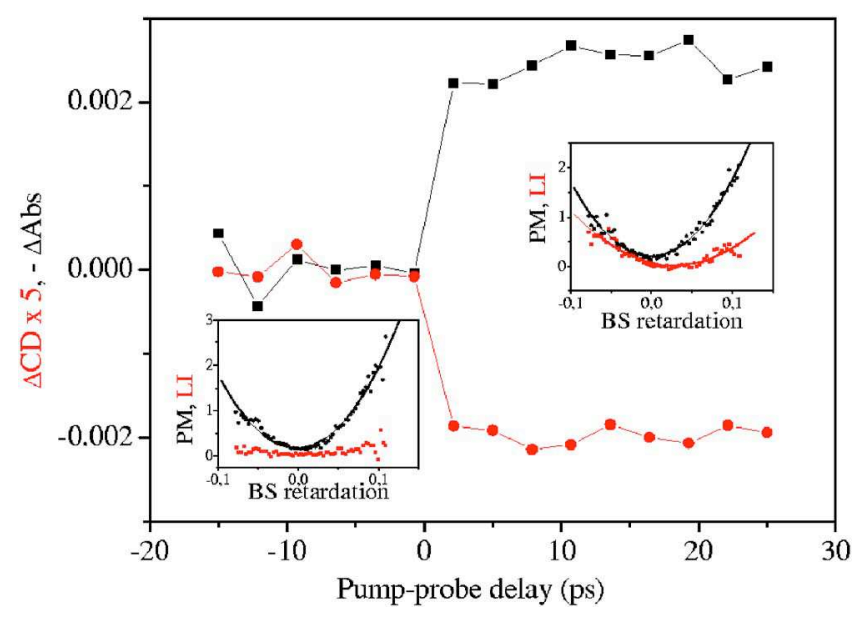

Fig. 4. (Color online) Pump-induced absorption change (squares) and CD change (dots) in $\mathrm{MbCO}$ at $265 \mathrm{~nm}$ as a function of the pump-probe delay. The sign of $\Delta \alpha$ has been changed for the sake of clarity. The insets show the raw PM and LI data for a negative and a positive delay.

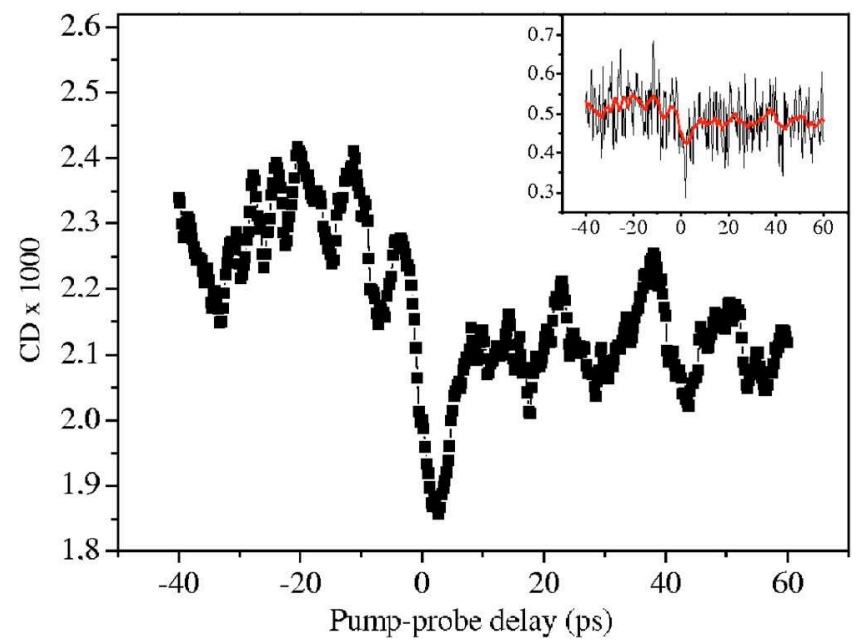

Fig. 5. (Color online) Time-resolved CD experiment in $\mathrm{MbCO}$ at $265 \mathrm{~nm}$ obtained by modulation of the probe polarization by a Pockels cell. Average of 20 curves and smoothing have been carried out. The inset shows the data prior to smoothing.

$440 \mu \mathrm{M}, \mathrm{pH}=8.0 \mathrm{MbCO}$ solution placed in a $1 \mathrm{~mm}$ thick silica cell. The cell is continuously rotated so that a fresh sample is available at each laser shot. Photodissociation of CO is initiated by a $400 \mathrm{~nm}, 200 \mathrm{fs}, 320 \mathrm{~nJ}$ pulse.

In Fig. 4, we have plotted $\Delta \alpha$ and $\Delta \eta$ for $\mathrm{MbCO}$ as a function of the pump-probe delay. The insets show the corresponding raw data. Both curves display a stepwise behavior, showing that equilibration to steady-state $\mathrm{Mb}$ is not resolved with our $1 \mathrm{ps}$ time resolution, in agreement with the dynamics known from visible experiments. ${ }^{11}$ The amplitude of the absorption change indicates that only a few percent of $\mathrm{MbCO}$ molecules are photoexcited. The measured CD change is $4 \pm 0.3 \times 10^{-4}$ corresponding to the passage from $\mathrm{MbCO}$ to $\mathrm{Mb}$ (cf. Fig. 3). For the CD signal as well as for the absorption one, no intermediate species is observable between the $\mathrm{MbCO}$ and $\mathrm{Mb}$ steady states. This latter measurement can be compared with a direct measurement performed with the polarization modulation technique (Fig. 5). This curve is obtained with the 
technique developed in Ref. 3 . Figure 5 represents the average of 20 curves. After smoothing, it clearly displays a CD change. These results are in perfect agreement with Fig. 4. Comparison of these two figures allows us to discuss the advantages of the two techniques. Actually, one sees that the CD curve in Fig. 4 is smoother than the one in Fig. 5 even though the acquisition time for the latter was approximately ten times longer than for the former one. This shows that the new technique is much more user friendly than the previous one, especially for weak CD changes. The only advantage of the previous technique is that it allows for a more precise measurement of the steady-state CD. However, steady-state CD spectra are easily measurable with CD spectrophotometers, and it is more useful to have a precise and rapid measurement of the pump-induced CD change.

In Fig. 5, one sees a peak in the CD curves at the zero delay. We have therefore conducted our experiment by focusing around the zero delay. Results are displayed in Fig. 6. We recover the strong peak. This peak is due to overlap of the pump and the probe. It allows the pumpprobe cross-correlation time to be measured to $800 \mathrm{fs}$. As apparent in the inset, at the peak, the signal is so strong that the approximate treatment of Section 1 is no longer valid. It is not clear whether this peak is a pure artifact due to the pump-probe overlap or the signature of the excited state of the heme, which is known to be very short lived. ${ }^{12}$ From a practical point of view, it allows us to ascertain the subpicosecond time resolution of our setup.

\section{Pump-Induced Optical Rotation}

We have taken advantage of the versatility of our technique to measure the pump-induced OR. Results are displayed in Fig. 7. Although very weak $\left(\Delta \delta=-5.6 \times 10^{-4}\right)$, an OR change is clearly measurable. Very little is known about $\mathrm{MbCO}$ OR. OR is expected to vanish when CD is maximum, but it is very probable that this OR at $265 \mathrm{~nm}$ is actually related to the strong absorption bands existing in the far UV (below $220 \mathrm{~nm}$ ). Existence of nonzero OR in hemoproteins is asserted in Ref. 13. This experiment

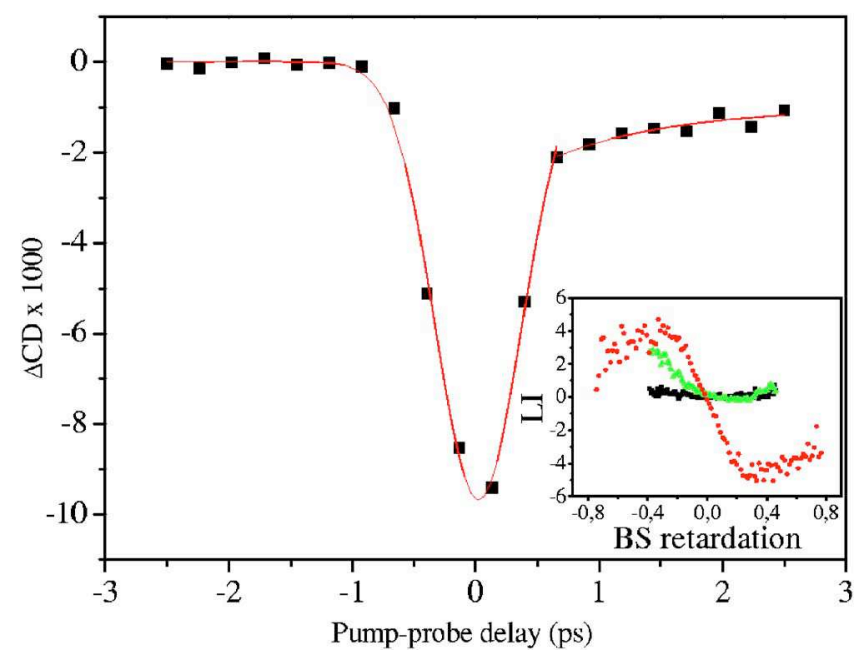

Fig. 6. (Color online) Pump-induced CD change around the zero delay. The inset shows three characteristic raw data (negative, zero, and positive delays).

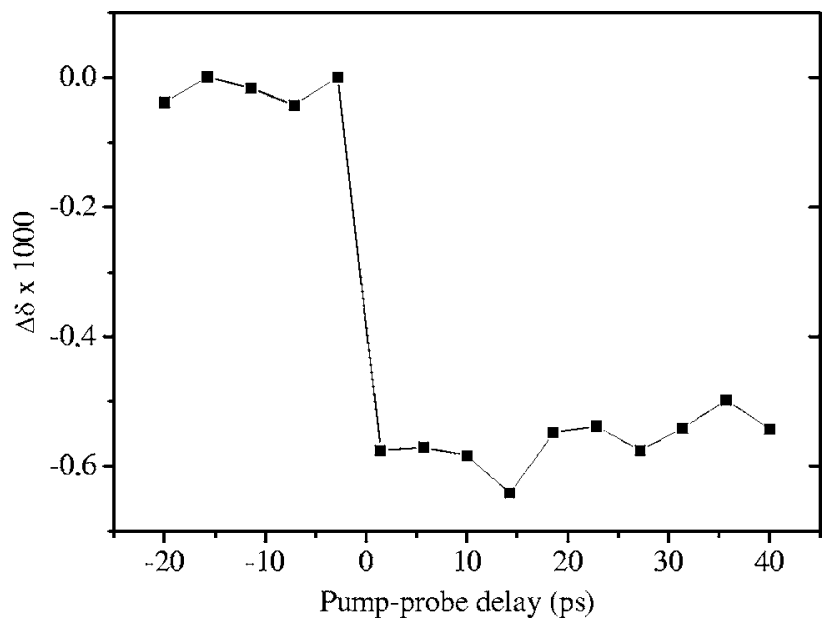

Fig. 7. Pump-induced OR change in $\mathrm{MbCO}$ at $265 \mathrm{~nm}$ as a function of the pump-probe delay.

shows that our technique allows for a complete determination of pump-induced optical activity in a simple way.

\section{CONCLUSION}

In this paper, we have proposed a new technique to measure pump-induced CD and OR in chiral molecules. This technique is based on the measurement of the change of ellipticity induced by the pump. Development of the calculation shows that the technique can strongly enhance the pump-induced CD and OR as soon as the absorption change is weak enough. We have brought experimental evidence of this assertion by measuring the change of CD in a chiral ruthenium complex. Symmetrical results are obtained for the two enantiomers as expected for chiral signals. We have also applied this technique to the changes of CD and OR in the UV following photodissociation of CO from myoglobin. Pump-induced CD and OR are readily obtained despite their extreme weakness. This techique should be very well fitted to the study of proteins in the UV where absorption changes are not expected to be very strong; whereas noticeable changes in CD could indicate conformational changes such as the modification of the secondary structure.

\section{APPENDIX A}

In this appendix, we want to consider the effects of linear birefringence (LB) and linear dichroism (LD) on the measurement of $\mathrm{OR}$ and $\mathrm{CD}$ changes. To do so, we consider the effect of LB alone on an achiral sample. The Jones matrix for $\mathrm{LB}$ is ${ }^{9}$

$$
M_{\mathrm{LB}}=\left[\begin{array}{cc}
e^{\mathrm{i} b} \cos ^{2} \theta+e^{-\mathrm{i} b} \sin ^{2} \theta & \mathrm{i} \sin b \sin 2 \theta \\
\mathrm{i} \sin b \sin 2 \theta & e^{-\mathrm{i} b} \cos ^{2} \theta+e^{\mathrm{i} b} \sin ^{2} \theta
\end{array}\right] \text {, }
$$

where $\theta$ is the angle of the fast axis of the birefringent optics with respect to the probe polarization and $2 b$ is the 
retardation. If we take $\theta$ and $b \ll 2 \pi, M_{\mathrm{LB}}$ reduces to the lowest order to

$$
\left[\begin{array}{cc}
1 & 2 \mathrm{i} b \theta \\
2 \mathrm{i} b \theta & 1
\end{array}\right],
$$

and it is straightforward to calculate the electric field after the BS and the analyzer at an angle $\varepsilon$ compared to the crossed position as

$$
E_{\mathrm{LB}}^{\text {out }}=\left[\begin{array}{c}
1 \\
\varepsilon+\mathrm{i}(X+2 b \theta)
\end{array}\right] .
$$

Comparing this equation with Eq. (7), one sees that the effect of birefringence is indiscernible from CD. A calculation of the effect of LD is immediately obtained from this calculation by replacing $b$ by i $\beta$ where $2 \beta$ is the absorption difference for the two polarization directions, and we obtain

$$
E_{\mathrm{LD}}^{\text {out }}=\left[\begin{array}{c}
1 \\
(\varepsilon+2 \beta \theta)+\mathrm{i} X
\end{array}\right],
$$

which shows that LD is indiscernible from OR. It is noteworthy that $\mathrm{LB}$, a refractive index effect, is similar to CD, an absorptive effect, and similarly that LD is similar to OR. In the case of pump-induced LB or LD, $\theta$ is the angle between the pump and the probe polarizations, and these effects will interfere with the pump-induced CD or OR.

F. Hache's e-mail address is francois.hache @polytechnique.edu.

\section{REFERENCES}

1. J. W. Lewis, R. F. Tilton, C. M. Einterz, S. J. Milder, I. D. Kuntz, and D. S. Kliger, "New technique for measuring circular dichroism changes on a nanosecond time scale. Application to (carbonmonoxy)myoglobin and (carbonmonoxy)hemoglobin,” J. Phys. Chem. 89, 289-294 (1985).

2. X. Xie and J. D. Simon, "Picosecond time-resolved circular dichroism study of protein relaxation in myoglobin following photodissociation of CO," J. Am. Chem. Soc. 112, 7802-7803 (1990).

3. T. Dartigalongue and F. Hache, "Observation of sub-100 ps conformational changes in photolyzed carbonmonoxymyoglobin probed by time-resolved circular dichroism," Chem. Phys. Lett. 415, 313-316 (2005).

4. T. Dartigalongue and F. Hache, "Classical calculation of myoglobin circular dichroism spectrum: simulation of a time-resolved experiment," J. Chem. Phys. 123, 184901 (2005).

5. S. C. Bjorling, R. A. Golbeck, S. J. Milder, C. A. Randall, J W. Lewis, and D. S. Kliger, "Analysis of optical artifacts in ellipsometric measurements of time-resolved circular dichroism," J. Phys. Chem. 95, 4685-4694 (1991).

6. T. Dartigalongue and F. Hache, "Precise alignment of a longitudinal Pockels cell for time-resolved circular dichroism experiments," J. Opt. Soc. Am. B 20, 1780-1787 (2003).

7. N. Sreerama and R. W. Woody, "Circular dichroism of peptides and proteins," in Circular Dichroism. Principles and Applications, 2nd ed., N. Berova, K. Nakanishi, and R. W. Woody, eds. (Wiley-VCH, 2000), pp. 601-620.

8. C. Brosseau, Fundamentals of Polarized Light (Wiley, 1998).

9. X. Xie and J. D. Simon, "Picosecond circular dichroism spectroscopy: a Jones matrix analysis,” J. Opt. Soc. Am. B 7, 1673-1684 (1990).

10. H. Graener, G. Seifert, and A. Laubereau, "Direct observation of rotational relaxation times by time-resolved infrared spectroscopy," Chem. Phys. Lett. 172, 435-439 (1990).

11. J. W. Petrich, C. Poyart, and J. L. Martin, "Photophysics and reactivity of heme proteins: a femtosecond absorption study of hemoglobin, myoglobin and protoheme," Biochemistry 27, 4049-4060 (1988).

12. S. Franzen, L. Kiger, C. Poyart, and J.-L. Martin, "Heme photolysis occurs by ultrafast excited state metal-to-ring charge transfer," Biophys. J. 80, 2372-2385 (2001).

13. D. W. Urry, "The heme chromophore in the ultraviolet," J. Biol. Chem. 242, 4441-4448 (1967). 